CIRJE-F-697

\title{
A Survey on Modeling and Analysis of Basis Spreads
}

\author{
Masaaki Fujii \\ Graduate School of Economics, University of Tokyo
}

Akihiko Takahashi

University of Tokyo

December 2009; Revised in February 2012

CIRJE Discussion Papers can be downloaded without charge from:

http://www.cirje.e.u-tokyo.ac.jp/research/03research02dp.html

Discussion Papers are a series of manuscripts in their draft form. They are not intended for circulation or distribution except as indicated by the author. For that reason Discussion Papers may not be reproduced or distributed without the written consent of the author. 


\title{
A Survey on Modeling and Analysis of Basis Spreads *
}

\author{
Masaaki Fujii ${ }^{\dagger}$ Akihiko Takahashi ${ }^{\ddagger}$
}

\begin{abstract}
The recent financial crisis has spiked the credit and liquidity premia among financial products, and significant widening of basis spreads among Libors with different tenors and currencies has been observed in interest rate markets. Our previous work, "A Note on Construction of Multiple Swap Curves with and without Collateral" has developed an arbitrage-free curve construction method with all the relevant spreads taken into account. This short note carries out a brief survey on the existing analysis of spreads' dynamics and pricing models as a preparation for the development of a model that enables us to price and hedge generic financial derivatives under the new market condition.
\end{abstract}

Keywords : Libor, swap, tenor, swap spread, curve, overnight index swap, cross currency, basis spread

*This research is supported by CARF (Center for Advanced Research in Finance) and the global COE program "The research and training center for new development in mathematics." The authors are not responsible or liable in any manner for any losses and/or damages caused by the use of any contents in this research.

${ }^{\dagger}$ Graduate School of Economics, The University of Tokyo

${ }^{\ddagger}$ Graduate School of Economics, The University of Tokyo 


\section{Introduction}

The recent financial crisis and the associated credit/liquidity crunch have caused a significant widening and higher volatilities among various basis spreads ${ }^{1}$ in the world of interest rate markets. As a response to this market development, construction of more sophisticated financial models that enable us to price and hedge generic financial products consistently in the presence of various basis spreads has risen a great attention among market participants recently. In the previous work, "A Note on Construction of Multiple Swap Curves with and without Collateral" [1], we have developed the method to construct a multiple swap curves consistently with all the relevant basis spreads. Using the method we have explained, one can price and hedge interest rate swaps (IRS), tenor swaps (TS) ${ }^{2}$, and cross currency swaps (CCS) consistently in no-arbitrage way.

The important remaining task is to construct an interest rate model and its simulation scheme with dynamic basis spreads. This note carries out a brief survey of major existing works about the spread dynamics and pricing models in order to gather the information about the features that the model needs to facilitate. In the next section, we will review econometric analysis of swap spreads, and then move to the several proposed pricing models.

\section{Review of econometric analysis on the spread dynamics}

As explained in the previous work [1], the important ingredients of swap curve construction are Libor-OIS, CCS, and TS basis spreads ${ }^{3}$. Although we could not find any specific research on the term structure and/or dynamics of these basis spreads, there exist a great amount of studies carried out on the swap spreads, especially for the US market. Swap spread represents the interest rate differentials between the swap rate and the government bond par yield with the same maturity. Since they reflect, among others, the default risk and liquidity differentials between these two instruments, we can expect that they have something important common with the spreads that is now interesting to us.

Traditionally, the credit worthiness of counter parties has been taken as the primary factor driving the swap spreads, and indeed there exist a huge number of academic works adopting this assumption. Although it may have been reasonable at the early stage of swap markets, the current industry practice that the both parties enter a swap contract with a netting and collateral agreements should eliminate ( in a significant way if not completely ) the counter party default risk, as we have emphasized in Sec.3 of Ref. [1]. Furthermore, even without the collateral agreement, the research of Duffie and Huang (1996) [2] has demonstrated that the effect of the counter party credit risk on the IRS rate is extremely small ${ }^{4}$.

In fact, the new trend of economic studies on the swap spreads takes the absence of

\footnotetext{
${ }^{1}$ In general, a basis spread means the interest rate differential between two different floating rates.

${ }^{2}$ It is a floating-vs-floating swap that exchanges Libors with two different tenors with a fixed spread in one side.

${ }^{3}$ OIS stands for the overnight index swap, which exchanges floating stream of daily compounded overnight rates, and the fixed coupon stream.

${ }^{4}$ Interestingly, their results suggest that there seems non-negligible impact on the spread of CCS due to the high volatility of foreign exchange rates and an exchange of principals, if there is no collateral posting.
} 
the counter party default risk as granted, and has shifted its emphasis on the credit risk embedded in Libor itself and liquidity factor in government bonds. Grinblatt (2001) [3] has assumed that the Libor is totally risk free in addition to the absence of the counter party credit risk. He introduced the convenience yield for holding a government bond due to its liquidity advantage. It is modeled as $y=\beta r+x$, where $\beta$ is a constant, $r$ is the instantaneous risk-free rate, and $x$ is a some state variable. He has argued that the investment in a government bond pays the instantaneous dividend $y$, and then identifies the yield converted from the present value of its payment stream as the swap spreads. He used a one factor affine model both for the $r$ and $x$ and has found reasonable fit to the observed swap spreads.

He (2001) [4] has considered that the short-term financing spread and its future expectation play the key role to determine the term structure of swap spreads. If there is no counter party credit risk, the long position of the government bond and the receiver position of the swap are basically equivalent except how their positions are being financed, i.e., the former requires the general collateral (GC) repo rate, and the latter requires the Libor rate. He has attributed the existence of swap spreads to a compensation for the short-term financing spreads between the Libor rate and the term GC-repo rates.

More specifically, he considered the following dynamics for the instantaneous interest rate:

$$
\begin{aligned}
d x_{1}(t) & =\left[k_{1}\left(\bar{x}_{1}-x_{1}(t)\right)+\lambda_{1} \sigma_{1}\right] d t+\sigma_{1} d w_{1}^{*} \\
d x_{2}(t) & =\left[k_{2}\left(\bar{x}_{2}-x_{2}(t)\right)+\lambda_{2} \sigma_{2}\right] d t+\sigma_{2} d w_{2}^{*} \\
d r(t) & =\left[k_{r}(\Theta(t)-r(t))+\lambda_{r} \sigma_{r}\right] d t+\sigma_{r} d w_{r}^{*}
\end{aligned}
$$

where $\Theta(t)=x_{1}(t)+x_{2}(t)$, and the other coefficients are constants. $w_{i}^{*}$ denotes the independent Brownian motion under the equivalent probability measure $P^{*}$, which is related to the physical measure $P$ by the relation of $w_{i}^{*}=w_{i}-\lambda_{i} t$. By adjusting $k_{1}$ and $k_{2}$ suitably $\left(k_{1}<<k_{2}\right)$, we can make $x_{1}$ determine the overall interest rate level and $x_{2}$ the slope. He borrowed the most of the parameter from the existing works, and has obtained $\left(x_{1}(0), x_{2}(0), r(0), \lambda_{1}\right)$ by calibration to the historical data. Specifically, he has fitted the model to FF target rate (as the proxy of GC-repo rate), 2yr and 10yr Treasuries using $r(0), x_{1}(0)$ and $x_{2}(0)$, and also used the 30yr Treasury to get the risk premium $\lambda_{1}$ for holding long-term government bonds.

He also defined the financing spread as $\delta(t)=R(t)-r(t)$, where $R(t)$ denotes the instantaneous rate corresponding to the Libor. The dynamics of $\delta$ is specified as

$$
\begin{aligned}
d \delta_{1}(t) & =\left[k_{\delta_{1}}\left(\bar{\delta}_{1}-\delta_{1}(t)\right)+\lambda_{\delta_{1}} \sigma_{\delta_{1}}\right] d t+\sigma_{\delta_{1}} d w_{\delta_{1}}^{*} \\
d \delta_{2}(t) & =\left[k_{\delta_{2}}\left(\bar{\delta}_{2}-\delta_{2}(t)\right)+\lambda_{\delta_{2}} \sigma_{\delta_{2}}\right] d t+\sigma_{\delta_{2}} d w_{\delta_{2}}^{*} \\
d \delta(t) & =\left[k_{\delta}\left(\bar{\delta}_{1}+\bar{\delta}_{2}-\delta(t)\right)+\lambda_{\delta} \sigma_{\delta}\right] d t+\sigma_{\delta} d w_{\delta}^{*}
\end{aligned}
$$

where the notations are fixed as similarly as $r$, and all the Brownian motions are assumed to be independent. As for the historical data, he used 1-month Libor, 2yr, 10yr and 30yr swap information to fix $\left(\delta_{1}(0), \delta_{2}(0), \delta(0), \lambda_{\delta_{1}}\right)^{5}$.

\footnotetext{
${ }^{5}$ Assuming suitable relations among $k_{\delta_{1}}, k_{\delta_{2}}$ and $k_{\delta}$, the dynamics of $R$ turns out to be the same form and it is possible to obtain a simple analytical form for the swap rate.
} 
Using the above model, he has studied the behavior of fitted parameters between 1998 and 2000. The result shows that both of the short and long-term factors of swap rates are higher than those of Treasuries, which corresponds to positive values of $\delta(0)$ and $\delta_{1}(0)$. As for the size of contribution to the swap spread, the long-term factor is more important. The long-term risk premium of swaps is lower than that of Treasuries in most of the studied period, but turns higher around the start of 2000, which corresponds to the time when the US Treasury Department announced its Treasury buy-back program, which increases the demand for long-term bonds. Another interesting finding is about the slope factor $\delta_{2}(0)$. The spread slope factor is always negative, which basically means a steeper swap curve than that of Treasuries, or the upward slope of the swap spread. He compared the fitted results using the on-the-run and the off-the-run Treasuries, and showed that the size of the spread slope factor is meaningfully smaller when using the off-the-run Treasuries although there is no significant changes in other factors. It suggests that the upward slope of the swap spread has a close link to the liquidity advantage for holding the on-the-run special Treasuries.

Although it is clear that both of the credit risk and liquidity premium (or convenience yield) are playing critical roles to determine swap spreads, it is not obvious how to distinguish these two and their relative importance in these works. The possible way is to perform joint calibration with corporate bonds to extract the credit component. Regarding to this line of arguments, one noteworthy fact was pointed out by Collin-Dufresne and Solnik (2001) [5]. Since the Libor is the interest rate for the top-quality banks, it has been assumed that the credit component embedded in the Libor is equivalent to that of Libor-bonds, which are fixed-coupon bonds negotiated OTC and issued by top-quality corporates (usually banks and financial institutions) rated AA or better. However, they have emphasized that Libor-bond yields are non-negligibly higher than the corresponding swap rates, and the spread (they called it Libor-swap spread) increases with maturity. Typical size of the Libor-swap spread is around 15bp for the period studied. They adopted the socalled reduced-form model discussed by Duffie and Singleton (1999) [6], but have extended the model so that they could incorporate the difference in credit quality between Libor, whose credit quality is constantly refreshed, and a top-rated issuer who may experience downgrades within the life of its bonds. Specifically, they have considered the following simple model of credit spread for an issuer who is top-rated at time $t$ as $(s \geq t)$

$$
\begin{aligned}
d \delta^{t}(s) & =\kappa_{\delta}(s)\left[\bar{\delta}^{t}(s)-\delta^{t}(s)\right] d s+\sigma_{\delta}(s) d w_{\delta}(s)+\nu_{1}(s) d N^{t}(s) \\
d \bar{\delta}^{t}(s) & =\nu_{2}(s) d N^{t}(s) .
\end{aligned}
$$

Here, deterioration in credit quality is triggered by a point process with deterministic intensity $\lambda^{t}(s)$ and associated counting process $N^{t}(s)\left(N^{t}(t)=0\right): N^{t}(s)$ is equal to the number of jumps in credit quality between $t$ and $s$ and gives rise to the widening of both the short- and long-term credit spreads. They have shown that the possible downgrade of issuer plays an dominant role to explain the Libor-swap spreads.

Following these works, Feldhütter and Lando (2008) [7] have recently carried out the detailed analysis to decompose the swap spreads into three components: a convenience yield for holding Treasuries; a credit spread arising from the credit risk element in Libor; and a swap specific residual component. They have constructed a joint pricing model for Treasuries, corporate bonds, and swap rates using six latent variables. Particularly, they 
have set up a rating-based model to consistently separate the AA credit component from the change of a credit spread due to the possible rating migrations.

The vector of latent variables consists of six independent affine processes:

$$
\begin{aligned}
X_{t} & =\left(X_{1 t}, \cdots, X_{6 t}\right)^{\prime} \\
d X_{i t} & =k_{i}\left(X_{i t}-\theta_{i}\right) d t+\sqrt{\alpha_{i}+\beta_{i} X_{i t}} d W_{i}^{P}
\end{aligned}
$$

where, $\left\{W_{i}^{P}\right\}$ are independent Brownian motions under the physical measure $P$. The processes under the equivalent martingale measure $\mathrm{Q}$ are given by

$$
d X_{i t}=k_{i}^{*} X_{i t} d t+\sqrt{\alpha_{i}+\beta_{i} X_{i t}} d W_{i}^{Q}
$$

where $k_{i}^{*}=k_{i}-\lambda_{i} \beta_{i}$, and $\lambda_{i}=-k_{i} \theta_{i} / \alpha_{i}{ }^{6}$. Here, the means of $\left\{X_{i}\right\}$ under the Qmeasure are normalized to be zero. Using these variables, they specified the short rate of government bond and the risk-free rate as

$$
\begin{aligned}
r^{g}(X) & =a+X_{1}+X_{2}, \\
r(X) & =a+X_{1}+X_{2}+\left(e+X_{5}\right),
\end{aligned}
$$

where $a$ and $e$ are constants, and the factor $\left(e+X_{5}\right)(\geq 0)$ explains the convenience yield (or the liquidity premium) relative to the government bonds. Both the risk-free and the government bond allow closed form expressions. Finally, they have defined

$$
\mu(X)=b+X_{3}+X_{4}+c\left(X_{1}+X_{2}\right),
$$

for the description of credit parts, where both $b$ and $c$ are constants. Following Lando (1998) [8], they have specified the default intensity of a firm which is in rating category "i" at time $t$ as

$$
\lambda(X, i)=\nu_{i} \mu(X),
$$

where $\nu_{i}$ is a constant. The transition intensity from category " $\mathrm{i}$ " to category " $\mathrm{j}$ " is assumed to be

$$
a_{i j}(X)=\lambda_{i j} \mu(X)
$$

where $\lambda_{i j}$ is a constant matrix. They have estimated the matrix using the Moody's cooperate bond default database. Under these assumptions, the zero-coupon cooperate bond price has a closed form expression.

In order to capture the swap specific factor, such as the demand from hedging needs, the Libor is described with an additional factor to the credit component

$$
L(t, t+\tau)=\frac{1}{\tau}\left(\frac{1}{v^{L I B}(t, t+\tau)}-1\right),
$$

where

$$
v^{L I B}(t, t+\tau)=E_{t}^{Q}\left[\exp \left(-\int_{t}^{t+\tau}\left(r\left(X_{s}\right)+\nu_{A A}\left(X_{s}\right)+S\left(X_{s}\right)\right) d s\right)\right]
$$

\footnotetext{
${ }^{6}$ In order to keep the affine property, the risk premium is assumed to be proportional to the corresponding standard deviation.
} 
and

$$
S(X)=d+X_{6}
$$

is the residual component to capture the swap specific factor.

In a joint calibration, they have used the data of US Treasuries, swap rates, and corporate yields for the rating categories AAA, AA, A, and BBB on a weekly basis from the end of 1996 to the end of 2005. They found that by far the largest component of swap spreads comes from the convenience yield for owning Treasuries, which shows the assumption in Grinblatt (2001) is not unreasonable. Treasury yield is significantly lower than the risk-free rate, and the estimated risk-free rate quite closely tracks topquality corporate bond yields. However, they also found that the credit component is not negligibly small and that it typically increases with maturity. Their results show that the size of contribution from the credit component is quite stable. This is in a good contrast to the similar researches that attributes the difference between GC-repo and Libor rates to the credit component, which is a popular assumption but gives rise to much more volatile behavior of the credit contribution in the swap spreads (See, for example, Liu, Longstaff and Mandell (2006) [9].). The swap specific factor turned out to be significantly negative and pushed down the swap spread towards the end of 2000, which coincides the period where there was a significant MBS refinancing activity ${ }^{7}$.

\section{Review of existing pricing models in the presence of spreads}

Now, let us turn our attention to the existing works regarding to the pricing of derivatives in the presence of various basis spreads. We have found that there exist only a few works on the pricing models, and none of them treats all the swaps i.e., IRS, TS and CCS in consistent manner with no-arbitrage conditions.

Although Ametrano and Bianchetti (2009) [10] explains the detailed bootstrapping methodology, the resulting curves allow arbitrage. In order to recover the observed level of swap rates with different tenors, they simply construct a curve for each tenor following the same method explained in Sec.2.1 of Ref. [1]. For the tenor which does not have the direct quote as a fixed-vs-floating swap, they have used TS basis to calculate the implied swap rate and has followed the usual bootstrapping technique ${ }^{8}$. Their method results in multiple discounting curves within a single currency, which leads to the arbitrage possibility. In fact, as one can easily imagine, their method can recover the market price of tenor swap only when they use the different discounting curve for each leg ${ }^{9}$. They have not discussed the cross currency basis issues, either.

In the following work, Bianchetti (2008) [11] has tried to solve the above mentioned arbitrage problem by making use of the analogy of a FX model. He has continued to assume that they have a set of yield curves $\left\{C_{\tau}\right\}$ for every tenor $\tau$. As in the previous

\footnotetext{
${ }^{7}$ Refinancing of MBS causes the shortening its duration, which makes the investors in MBS securities adjust the interest rate hedge positions and leads to a significant demand for receiver swaps.

${ }^{8}$ For example, they simply subtract $3 \mathrm{~m} / 6 \mathrm{~m}$ TS basis to the swap quotes of fixed-vs-6mLibor IRS to imply out the $3 \mathrm{~m}$ tenor IRS quotes.

${ }^{9}$ Although they have described the method as if it is the market standard technique, we knows that it is not the case, at least in some of the biggest investment banks. It may not be too difficult to exploit the problem by making use of forward starting swaps.
} 
model, each yield curve contains the set of "discounting factor" related to the Libor as

$$
L(t, t+\tau)=\frac{1}{\delta(\tau)}\left(\frac{1}{P_{\tau}(t, t+\tau)}-1\right)
$$

where $\delta(\tau)$ is the appropriate day count faction. He also constructs the discounting curve $C_{d}$ that consists of a set of $\left\{P_{d}(t, T)\right\}$ being used as a discounting factor in the usual sense. Calibration instruments for $C_{d}$ are chosen from the liquidity considerations. He now assumes that all the payments in the market are discounted by this unique discounting curve $C_{d}$. Specifically, the present value of the future Libor payment is calculated as

$$
\begin{aligned}
P V(t) & =E_{t}^{Q}\left[e^{-\int_{t}^{T} r(s) d s} \delta(\tau) L(T-\tau, T)\right] \\
& =E_{t}^{Q}\left[e^{-\int_{t}^{T} r(s) d s}\left(\frac{1}{P_{\tau}(T-\tau, T)}-1\right)\right] \\
& =P_{d}(t, T) E_{t}^{Q_{T}}\left[\frac{1-P_{\tau}(T-\tau, T)}{P_{\tau}(T-\tau, T)}\right],
\end{aligned}
$$

where $E^{Q_{T}}[]$ denotes the expectation under the forward measure where $P_{d}(\cdot, T)$ is used as a numeraire. Note that

$$
F_{\tau}(t, T-\tau, T) \neq E_{t}^{Q_{T}}\left[\frac{1-P_{\tau}(T-\tau, T)}{P_{\tau}(T-\tau, T)}\right]
$$

where the "forward" $F_{\tau}$ is defined as

$$
F_{\tau}(t, T-\tau, T)=\frac{1}{\delta(\tau)}\left(\frac{P_{\tau}(t, T-\tau)}{P_{\tau}(t, T)}-1\right)
$$

since the numeraire used is not $P_{\tau}(\cdot, T)$ but $P_{d}(\cdot, T)$. He models it as a quanto, where the payment currency and Libor currency are different (but with a constant FX rate $(=1)$ ). Once we note that the framework is basically equivalent to the multi-currency model with a pegged FX rate, it is easy to understand that there is no arbitrage possibility ${ }^{10}$.

We can calculate the relevant expectation values once we fix the underlying stochastic dynamics for $C_{d}, C_{\tau}$ and the correlation structure among them. Although this model can solve the arbitrage issue, we have to conclude that the method is not practical for the everyday pricing and hedging. First of all, we cannot separate the construction of $C_{\tau}$ from the underlying model calibration which also needs to be fitted to the option market for each tenor. This fact also complicates the delta hedging against the move of TS basis spreads. Unobservable nature of $C_{\tau}$ that consists of $\left\{P_{\tau}(t, T)\right\}$ gives rise to the most of these difficulties, and requires us to specify the IR model under the situation where there is no domestic swap market but the market of quanto swaps only ${ }^{11}$.

\footnotetext{
${ }^{10}$ When the spread comes from the credit risk, the calculation is very similar to the measure change between the survival and the risk-free measures explained in Schönbucher (2000) [12].

${ }^{11}$ It is worthwhile to note that the same framework is quite useful for certain type of exotics. For example, consider the product which involves the government bond yield and Libor as reference rates at the same time, and also assumes that the trade is not collateralized (and hence Libor funding) for simplicity. Due to the quite different natures of two markets especially from the funding perspective, it may be reasonable to construct the two curves $\left(C_{g o v}, C_{L i b}\right)$, from the government bond par yields and swap rates respectively,
} 
Kijima et al. (2008)[13] has discussed the way to construct curves for a single forward Libor and a government bond yield in addition to the unique discounting curve. They have assumed that the USD Libor is frictionless, and adopted the similar method which we have explained in Sec.2.2 of Ref. [1] to extract the set of discounting factors. After the estimation of forward rates, they calibrated a short-rate based model for each of the discounting rate, CCS basis spread and the government bond yield for a simple demonstration.

Recently, Mercurio (2008) proposed a practical simulation scheme under the presence of basis spreads in the framework of the market model. He directly models the dynamics of conditional Libor expectation under the forward measure of $C_{d}$ :

$$
L\left(t, T_{k-1}, T_{k}\right)=E^{T_{k}}\left[L\left(T_{k-1}, T_{k}\right) \mid \mathcal{F}_{t}\right],
$$

where $P_{d}\left(\cdot, T_{k}\right)$ is used as a numeraire. Here, $\{k\}$ denote the reset times of Libors. The risk-free forward rate is defined as usual by discount factors in $C_{d}$ :

$$
F\left(t, T_{k-1}, T_{k}\right)=\frac{1}{\delta_{k}}\left(\frac{P_{d}\left(t, T_{k-1}\right)}{P_{d}\left(t, T_{k}\right)}-1\right),
$$

where $\delta_{k}$ denotes the day count fraction between $T_{k-1}$ and $T_{k}$. Since both of these variables are martingales under this forward measure, we can define their dynamics as

$$
\begin{aligned}
& d L\left(t, T_{k-1}, T_{k}\right)=\sigma_{k}(t,\{L\}) \cdot d W^{T_{k}}(t) \\
& d F\left(t, T_{k-1}, T_{k}\right)=\sigma_{k}^{(d)}(t,\{F\}) \cdot d W^{T_{k}}(t) .
\end{aligned}
$$

Here, $W^{T_{k}}$ is the Brownian motion under this forward measure and assumed to be $d$ dimensional. Also $\sigma$ may depend on other state variables although they are not given explicitly.

In order to run the simulation, we would like to use a discretely-compounded money market measure, where the numeraire is defined by

$$
B_{d}^{\mathcal{T}}(t)=\frac{P_{d}\left(t, T_{\beta(t)-1}\right)}{\prod_{j=0}^{\beta(t)-1} P_{d}\left(T_{j-1}, T_{j}\right)},
$$

where $\beta(t)=m$ if $T_{m-2}<t \leq T_{m-1}$, and hence $t \in\left(T_{\beta(t)-2}, T_{\beta(t)-1}\right]$. Under this market measure $\mathcal{T}$, the dynamics of conditional Libor expectation is given by

$$
\begin{aligned}
d L_{k}(t) & =\sum_{j=\beta(t)}^{k} \frac{d\left\langle L_{k}, F_{j}\right\rangle_{t} \delta_{j}}{1+\delta_{j} F_{j}(t)}+\sigma_{k}(t) \cdot d W^{\mathcal{T}}(t) \\
& =\sum_{j=\beta(t)}^{k} \frac{\left(\sigma_{k}(t) \cdot \sigma_{j}^{(d)}(t)\right) \delta_{j}}{1+\delta_{j} F_{j}(t)} d t+\sigma_{k}(t) \cdot d W^{\mathcal{T}}(t),
\end{aligned}
$$

based on the method in Sec.2.1 of Ref. [1]. Their dynamics can be calibrated using the information of bond options and swaptions, with estimated correlation between them, say from historical data. Then, we can calculate the present value of a $C_{g o v}$-contingent payment by this pegged-FX framework, where we discount the cash flow by $C_{L i b}$ with appropriate quanto adjustments. The framework can be extended for more complicated deals. See also the related model in Kijima et al.(2008). However, as we have discussed, it does not seem to be practical to use the same framework for the TS basis spreads. 
and in the same way, we have

$$
d F_{k}(t)=\sum_{j=\beta(t)}^{k} \frac{\left(\sigma_{k}^{(d)}(t) \cdot \sigma_{j}^{(d)}(t)\right) \delta_{j}}{1+\delta_{j} F_{j}(t)} d t+\sigma_{k}^{(d)}(t) \cdot d W^{\mathcal{T}}(t)
$$

for risk-free forwards. Except the increased underlying processes, the procedures are completely parallel to the standard Libor market model.

Unfortunately, in the paper of Mercurio (2008), there exist several confusions about curve construction. He has not discussed the details of curve construction and has assumed the existence of $C_{d}$ and $C_{\tau}$ by referring to the work of Ametrano and Bianchetti (2009) [10]. He explains that $C_{\tau}$ is bootstrapped from the instruments with tenor $\tau$ and that there exist several choices for discount curves $C_{d}$, such as OIS, or combinations of liquid instruments. If we literally follow his suggestions, we cannot recover the market price of swaps although it is possible to construct an arbitrage free simulation system. They have not discussed a multi-currency situations nor the explicit implication to the pricing of generic derivatives in the presence of a collateral agreement due to the lack of understanding of consistent curve construction.

\section{Summary and implication}

As a summary, let us consider the implication of these works to the model setup for the generic derivative pricing under the presence of various spreads. From the econometric analysis, it seems quite likely that the spreads we are now dealing with, Libor-OIS, CCS and TS basis spreads, contain significant contributions from liquidity and a specific component of each instrument. In fact, it is easy to understand that the attempt to explain the TS basis spreads by adding the hazard rate on top of the risk-free rate will fail, since, in the absence of counter party default risk, adding the common hazard rate leads to only negligible TS spreads. Furthermore, it is most probable that there is a dominant contribution from the liquidity factor in CCS basis spread, otherwise we are forced to include unreasonably high credit risk among Libors and currencies of developed nations to explain the wide CCS basis in the market, even in the very short maturities. These considerations suggest us that we need to include a specific factor separately to each tenor of Libor and OIS to appropriately describe the actual market.

As for the pricing models, there exist only a few works pay attention to the importance of basis spreads. The model of Ametrano and Bianchetti (2009) [10] allows arbitrage, and its extended version with FX analogy in Bianchetti (2008) [11] has a technical difficulties to use it in actual trading although it escapes from the arbitrage possibility. The work of Mercurio (2008) [14], although it contains some confusions about the curve construction and lacks the discussion in a multi-currency situations, proposed an efficient simulation scheme in exactly parallel fashion to the Libor market model. It seems that the possible way is to use the set of expectations $\left\{E_{t}[L(T-\tau, T)]\right\}$, which is directly obtained after the curve construction following Ref. [1], as starting points of this model. The major advantage relative to the previous works is that we can separate the curve construction and option calibrations. 


\section{References}

[1] Fujii, M., Shimada, Y., Takahashi, A., 2009, "A note on construction of multiple swap curves with and without collateral," CARF Working Paper Series F-154, available at http://ssrn.com/abstract $=1440633$.

[2] Duffie, D., and M.Huang, 1996, "Swap rates and credit quality," Journal of Finance, 51, 921-949.

[3] Grinblatt, M., 2001, "An analytical solution for interest rate swap spreads," International Review of Finance 2(3), 113-149.

[4] He,H., 2001, "Modeling term structures of swap spreads," Working Paper, Yale School of Management.

[5] Collin-Dufresne, P., Solnik, B., 2001, "On the term structure of default premia in the swap and LIBOR markets," Journal of Finance 56, 1095-1114.

[6] Duffie, D., and K.Singleton, 1999, "Modeling term structures of defaultable bonds," Review of Financial Studies 12, 687-720.

[7] P.Feldhütter, D.Lando, 2008, "Decomposing swap spreads," Journal of Financial Economics, 88(2), 375-405.

[8] Lando, D., 1998, "On Cox processes and credit-risky securities," Review of Derivatives Research, 2, 99-120.

[9] Liu, J., Longstaff, F., Mandell, R., 2006, "The market price of risk in Interest rate swaps: the roles of default and liquidity risks," Journal of Business, 79(5), 2337-2359.

[10] Ametrano, F., Bianchetti, M., 2009, "Bootstrapping the illiquidity: Multiple yield curves construction for market coherent forward rates estimation," to be published in "Modeling Interest Rates: Latest advances for derivatives pricing," edited by F.Mercurio, Risk Books, 2009.

[11] Bianchetti, M., 2008, "Two curves, one price: Pricing and hedging interest rate derivatives using different yield curves for discounting and forwarding," Working paper.

[12] Schönbucher, P., 2000, "A Libor market model with default risk," Working paper.

[13] Kijima, M., K.Tanaka and T.Wong, 2008, "A multi-quality model of interest rates," Quantitative Finance.

[14] Fabio Mercurio, 2008, "Interest rate and the credit crunch: New formulas and market models," Working paper. 